\title{
ETM-IoT: Energy-Aware Threshold Model for Heterogeneous Communication in the Internet of Things
}

\author{
A. Vijaya Krishna ${ }^{1}$ and A. Anny Leema ${ }^{2, *}$ \\ ${ }^{1}$ School of Computer Science and Engineering, VIT University, Vellore, Tamilnadu, India \\ ${ }^{2}$ School of Information Technology and Engineering, VIT University, Vellore, Tamilnadu, India \\ "Corresponding Author: A. Anny Leema. Email: annyleema.a@vit.ac.in \\ Received: 09 March 2021; Accepted: 20 May 2021
}

\begin{abstract}
The internet of things (IoT) has a wide variety of applications, which in turn raises many challenging issues. IoT technology enables devices to closely monitor their environment, providing context-aware intelligence based on the real-time data collected by their sensor nodes. The IoT not only controls these devices but also monitors their user's behaviour. One of the major issues related to IoT is the need for an energy-efficient communication protocol which uses the heterogeneity and diversity of the objects connected through the internet. Minimizing energy consumption is a requirement for energyconstrained nodes and outsourced nodes. The IoT nodes deployed in different geographical regions typically have different energy levels. This paper focuses on creating an energy-efficient protocol for IoT which can deal with the clustering of nodes and the cluster head selection process. An energy threshold model is developed to select the appropriate cluster heads and also to ensure uniform distribution of energy to those heads and member nodes. The proposed model envisages an IoT network with three different types of nodes, described here as advanced, intermediate and normal nodes. Normal nodes are first-level nodes, which have the lowest energy use; intermediate nodes are second-level nodes, which have a medium energy requirement; and the advanced class are thirdlevel nodes with the highest energy use. The simulation results demonstrate that the proposed algorithm outperforms other existing algorithms. In tests, it shows a $26 \%$ improvement in network lifetime compared with existing algorithms.
\end{abstract}

Keywords: Internet of things; communication; energy; threshold; heterogeneous network

\section{Introduction}

In recent years, the utilization of smart devices in day-to-day life has increased rapidly. The 'internet of things' (IoT) enables objects such as mobile phones, radio frequency identification (RFID) tags, computers, sensors and other devices around us to communicate with each other [1-4]. IoT technology enables these devices to closely monitor the environment and provides context-aware intelligence based on the real-time data collected by their sensor nodes.

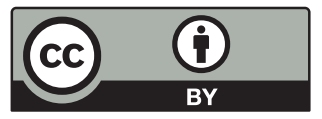

This work is licensed under a Creative Commons Attribution 4.0 International License, which permits unrestricted use, distribution, and reproduction in any medium, provided the original work is properly cited. 
The IoT not only controls the objects but also monitors their user's behaviour. The utilization of IoT is increasing rapidly in many applications such as environment monitoring, health monitoring, smart home models and others [5-8]. Hence, enhancing the performance of real-time data collection and transmission is a challenging task.

The main issues facing IoT sensor nodes are categorized as follows. The first is reliability. The lower layers of the network cannot assure the high reliability of the IoT; this needs to be addressed via the network layer-routing protocol. One of the solutions is to retransmit data packets, but this also increases the overhead on the transmission time and so affects performance [9]. The second challenge is how to improve the overall real-time performance of the IoT network. The final issue is to ensure that any routing protocol can provide a high degree of robustness, with each node in the network active and available for transmission [10]. The sensor nodes have limited battery power and computation capacity, so the protocol has to minimize the number of cluster heads [11-13].

These issues all need to be considered for designing new routing protocols. For the IoT, sensing the real-time environment is another challenge. The sensor nodes in the IoT may be in remote locations. Fig. 1 shows the different sensor nodes which may be deployed in different environments. These sensors continuously monitor their surroundings and may have limited flexibility to recharge their batteries. Many researchers have therefore worked on improving the performance of these nodes by decreasing their energy consumption. Certain algorithms have also been devised to help minimize transmission delays and energy usage [14,15].

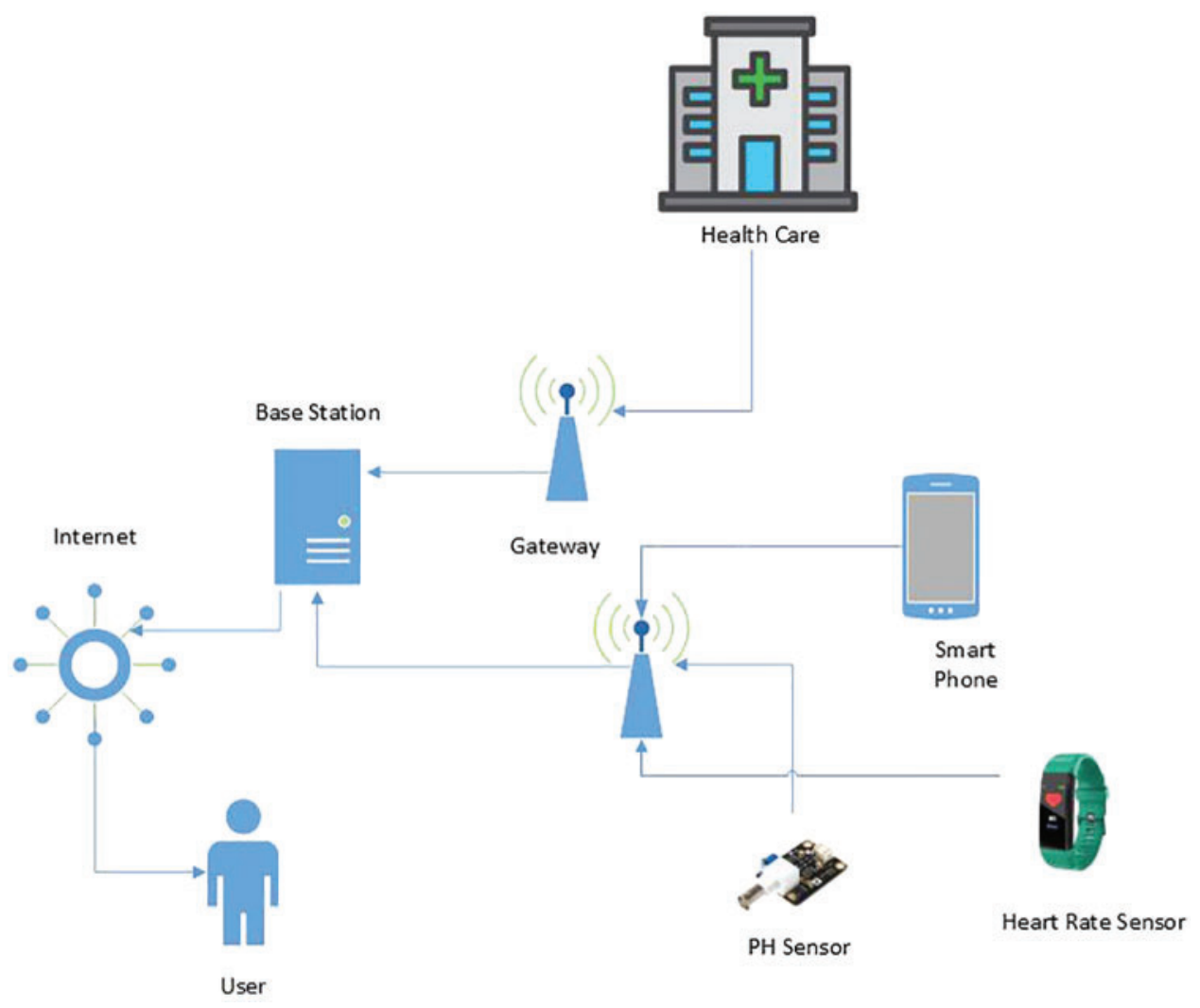

Figure 1: Sensor node deployment across the IoT network 
Clustering is a method considered by many researchers to minimize energy consumption across an IoT network. As the term suggests, nodes are grouped into clusters. Each cluster elects a cluster head to collect the data from other member nodes and to forward the data to a sink node. Therefore, the cluster head plays a prominent role in managing the amount of energy used.

Existing clustering algorithms are not yet sufficient to minimize the energy use of large-scale IoT networks. Fig. 2 shows a proposed heterogeneous network where all the nodes have unequal energy levels at the time of initialization. Sets of nodes with the lowest energy use are referred to here as 'normal' nodes. Those with the highest energy requirements are labelled 'advanced', while the energy use of the 'intermediate' nodes lies somewhere in between. The drawback of a homogenous network is that all nodes are initiated with the same energy level and so some typically die within a short space of time. The proposed heterogeneous network provides a better performance compared with the homogeneous network.

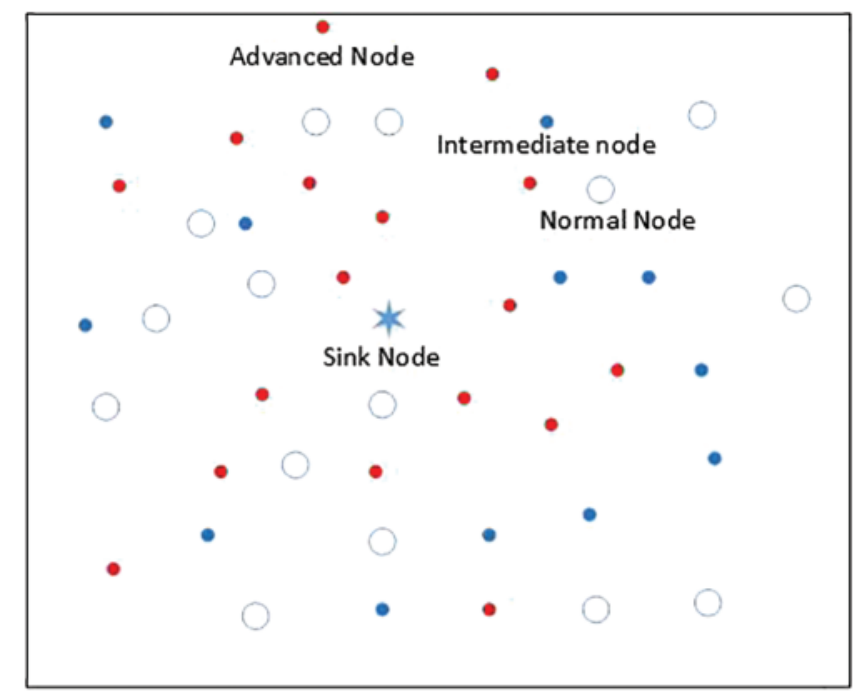

Figure 2: Proposed heterogeneous IoT network

In this paper, we focus on developing an algorithm for minimizing the energy consumption of the nodes and improving the lifetime of the network [16]. The rest of the paper is organised as follows. Section 2 deals with existing routing protocols used by IoT communication networks, as described in the literature. Section 3 explains the energy model for the proposed IoT network. Section 4 describes the energy threshold model for the network. Section 5 analyses the simulated environment. Finally, Section 6 concludes the research work.

\section{Literature Survey}

For the past decade, researchers have concentrated on developing a real-time routing mechanism for data transferred across the IoT. Most discussion has focused on energy efficiency in data transmission. In [17], the authors developed the LEACH protocol, where the cluster head is selected randomly. Therefore, each sensor node in the network has the opportunity to be selected as cluster head in every $1 / \mathrm{p}$ epochs. In further rounds, a random number is generated which lies in the range of 0 to 1 [18]. A node which has an energy level less than the threshold will act as a cluster head [19]. The next cluster head is selected from the set of non-cluster head 
nodes. After the cluster is formed, the cluster head transmits the ADV message to the member nodes. The nodes forward this data to the cluster head within the allocated time-division multiple access (TDMA) slot. The major drawback of the LEACH protocol is that it cannot be applied to large-scale sensing environments.

In a heterogeneous network, some advanced nodes have extra energy requirements compared with normal nodes [20]. The stable election process (SEP) algorithm is used to elect the cluster heads based on the weighted probabilities. It has the advantage of better performance and greater stability compared with the LEACH protocol. In [21], the authors propose a zoned stable election process (Z-SEP) algorithm, which is a hybrid model.

The Z-SEP contains three zones: 0,1 and 2. In Zone 0 , the nodes are distributed randomly. In Zones 1 and 2, the advanced nodes with high energy use are distributed equally and randomly. Two types of communication are used by the Z-SEP to transmit data to the base station. In the first, sensor nodes directly transmit the data to the base station. In the second, the sensor nodes send the data to the cluster head, which in turn forward the data to the base station. In each round, the cluster head is selected based on the threshold value.

\section{Energy Model for IoT Network}

In an IoT network, energy balancing and energy efficiency need to be considered as crucial parameters when designing the routing protocol. This paper concentrates on the development of a routing algorithm for minimizing the energy consumption of a network, thereby improving the lifetime of the nodes. To minimize energy dissipation, we considered the heterogeneity of a network on three different levels, based on the nodes' initial energy. All the nodes in the network are in statis. The normal nodes are the first level nodes which have lowest energy, the intermediate nodes are the second level nodes with medium energy and the advanced nodes are the third level nodes with highest energy.

Let the initial energy of normal nodes be represented by $\vartheta_{0}$, the energy of the advanced nodes be represented with $\vartheta_{0}(1+X)$ and the energy of the intermediate nodes be represented with $\vartheta_{0}(1$ + Y), Eqs. (1)-(3) representing the total energy of the normal, intermediate and advanced nodes.

$\vartheta_{N n}=k \vartheta_{0}(1-x-y)$

$\vartheta_{\text {In }}=k y \vartheta_{0}(1+Y)$

$\vartheta_{A n}=k x \vartheta_{0}(1+X)$

Here, the energies of the normal node are represented with $\vartheta_{N n}$, for intermediate nodes it is $\vartheta_{I n}$, and for advanced nodes it is $\vartheta_{A n}$. The advanced nodes in the network are represented as $x$ and their energy level is $X$. The intermediate nodes in the network are represented as $y$ and their energy level is given as $Y=X / 2$. $k$ represents the size of the nodes in each type. The total network energy consumption is given as

$$
\begin{aligned}
\vartheta_{t o t} & =k \vartheta_{0}(1-x-y)+k y \vartheta_{0}(1+Y)+k x \vartheta_{0}(1+X) \\
& =k \vartheta_{0}(1+x X+y Y)
\end{aligned}
$$


Here, we consider the SEP and LEACH protocol models for cluster head selection. For each type of node, the threshold value is calculated based on their probabilities of selecting the cluster head. Let $S_{1}, S_{2}$ and $S_{3}$ be the set of nodes belonging to each type and not selected as the cluster head of nodes to be selected as cluster head are given as follows.

For $\mathrm{N}_{\mathrm{n}}$ :

$$
\begin{aligned}
P_{N n} & =\frac{P}{1+x X+y Y} \\
T_{K_{N n}} & = \begin{cases}\frac{P_{N n}}{1-P_{N n}{ }^{\left(u \bmod 1 / P_{N n}\right)}} ; & \text { if } k_{N n} \in S_{1} \\
0 ; & \text { otherwise }\end{cases}
\end{aligned}
$$

For $\mathrm{I}_{\mathrm{n}}$ :

$$
\begin{aligned}
P_{I n} & =\frac{P(1+Y)}{1+x X+y Y} \\
T_{K_{I n}} & = \begin{cases}\frac{P_{I n}}{1-P_{I n}{ }^{\left(u \bmod 1 / P_{I n}\right)}} ; & \text { if } k_{I n} \in S_{2} \\
0 ; & \text { otherwise }\end{cases}
\end{aligned}
$$

For $A_{n}$ :

$$
\begin{aligned}
& P_{A n}=\frac{P(1+X)}{1+x X+y Y} \\
& T_{K_{A n}}= \begin{cases}\frac{P_{A n}}{1-P_{A n}{ }^{\left(u \bmod 1 / P_{A n}\right)}} ; & \text { if } k_{A n} \in S_{3} \\
0 ; & \text { otherwise }\end{cases}
\end{aligned}
$$

From Eqs. (5), (7) and (9), we can calculate the average probability of selecting the cluster heads per one iteration is given as

$k(1-x-y) P_{N n}+k x P_{A n}+k y P_{I n}=k P$

From Eq. (11), the value obtained for average probability of selecting cluster heads is equivalent to the value of the LEACH protocol in a heterogeneous environment.

The heterogeneous IoT network communication model is given in Fig. 3. A free space model or multipath fading model is used to calculate the energy dissipation in the heterogeneous network. Eqs. (12) and (13) shows the communication model where the sensor node consumes the energy for transmitting ' $n$ ' bits per packet.

$\vartheta_{T r}(n, d)=\vartheta_{T r \_e l e}(n)+\vartheta_{T r \_a m p}(n, d)$ 
$\vartheta_{T r}(n, d)= \begin{cases}\vartheta_{\text {ele }} \times n+\vartheta_{f s} \times n \times d^{2}, & d \leq d_{0} \\ \vartheta_{\text {ele }} \times n+\vartheta_{m p} \times n \times d^{4}, & d>d_{0}\end{cases}$

$\vartheta_{f s}$ and $\vartheta_{m p}$ denotes the parameters of the amplifier for transmitting the packets in free channel and multi path fading channel. $\vartheta_{\text {ele }}$ denotes the amount of energy consumed by the senor node to transmit the packet. The amount of energy to receive the packet is given as follows.

$\vartheta_{R c}(n)=\vartheta_{R c_{e} e l e}(n)+n \vartheta_{\text {ele }}$

The energy threshold value is computed for cluster head selection by estimating the energy consumption in each round. The proposed model computes the energy dissipation threshold value for all types of nodes to improve the network lifetime.

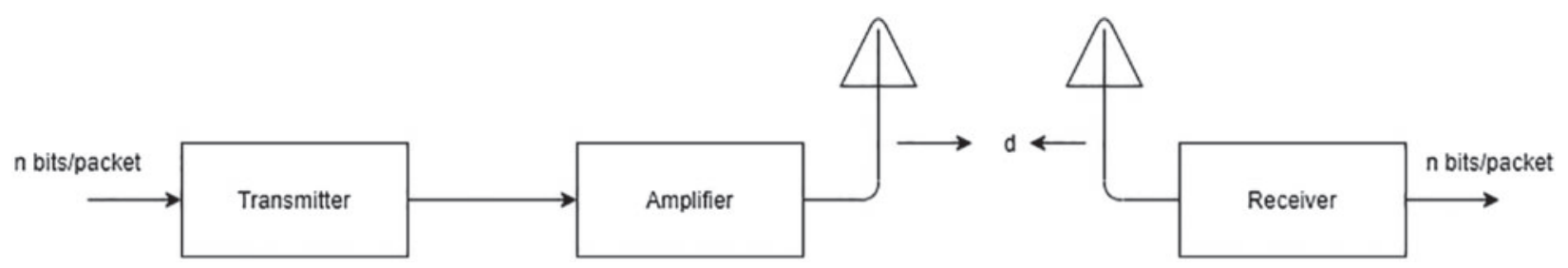

Figure 3: Communication model for IoT sensor nodes

\section{Proposed Algorithm}

As soon as the cluster head is elected, the nodes link to the cluster head based on the ADV message in the cluster. Once for each round, the cluster head and the nodes in the cluster will change. Nodes of any type which are near the sink node dissipate less energy than others for packet transmission. The clusters which are nearest to the sink node will continue with the same member nodes and cluster heads, without changing them in the next rounds.

The proposed algorithm deals with the threshold value for energy dissipation for all type of nodes in an IoT network. This threshold value will be the deciding factor for whether the cluster head and member nodes in each cluster continue to the next round. The cluster head residual energy is calculated after each round. If the residual energy value is less than the threshold value, then the network initiates the formation of new cluster along with a new cluster head election process. The proposed mechanism reduces the amount of energy dissipated for advertising the routing information and also for new cluster head formation.

The energy consumed by the cluster head and the sensor node will never be equal. The cluster head has some additional responsibilities such as data collection, aggregation and forwarding. Therefore, the cluster head will require more energy compared with the other sensor nodes. Hence, the proposed model allocates the high amplification energy to the cluster head and, in the next round, if the cluster head is changed to a normal node, the proposed model assigns low amplification energy to that particular node.

Let $\mathrm{q}$ be the total number of sensor nodes in the IoT network and $\mathrm{u}$ represent the percentage of clusters in the network. C represents the count of cluster head replacement. $\vartheta \mathrm{n}_{\operatorname{Tr}}$ represents the size of the packet to be transmitted and $\vartheta \mathrm{n}_{\mathrm{Rc}}$ represents the size of the packet to be received. 
CMC, 2022, vol.70, no.1

The energy spent for forming new cluster and cluster head replacement is given as $\vartheta_{\mathrm{C}}$ in Eq. (15).

$\vartheta_{C}=\left\{\vartheta_{n T r} \vartheta_{T r}+\vartheta_{n R c} \vartheta_{R c}(q u-1)\right\} C Q$

where $\mathrm{Q}=\mathrm{qu}$ denotes the cluster size, $\vartheta_{\mathrm{Tr}}$ represents the energy consumed for transmitting eight bits of data. $\vartheta$ Rc represents the energy consumed for receiving eight bits of data.

The cluster energy utilization is calculated by computing the supplied initial energy to all types of nodes (i.e., normal nodes, intermediate nodes and advanced nodes) within the cluster. $\vartheta_{Z}$ represents the energy utilization of the cluster, which is given as

$\vartheta_{Z}\left(N_{n}\right)=\vartheta_{0} \times q u$

$\vartheta_{Z}\left(I_{n}\right)=\vartheta_{0}(1-Y) \times q u$

$\vartheta_{Z}\left(A_{n}\right)=\vartheta_{0}(1-X) \times q u$

For a single round, the energy utilization of each cluster $\alpha$ is computed based on the node energy level in both conditions: one is the node acting as sensor node and another one is the node acting as cluster head.

$\vartheta_{C}(\alpha)=\left\{\left(Q_{\alpha}-1\right) \vartheta_{n T r} \vartheta_{T r} \times \vartheta_{n R c} \vartheta_{R c}\right\}+\left\{\left(Q_{\alpha}-1\right) \vartheta_{n T r} \vartheta_{T r}+\left(Q_{\alpha}-1\right) \vartheta_{n R c} \vartheta_{R c}\right\}$

The energy consumed by the sensor node to transmit the packets to the cluster head is represented with $\mathrm{q} \vartheta_{\mathrm{Tr}}$. The energy consumed for data aggregation at the cluster head side is given as $\mathrm{q}(\mathrm{Q}-1)_{\vartheta \mathrm{Rc}}$. The energy consumed for data forwarding by the cluster head to the sink node is given as $\mathrm{q}(\mathrm{Q}-1) \vartheta_{\mathrm{Tr}}$. The nodes move into a sleep state when there is no transmission or reception of data packets.

To calculate the energy threshold value for replacement of the cluster head, the number of rounds needs to be considered. $\mu$ calculates the total rounds for the normal, intermediate and advanced nodes as follows:

$\mu\left(N_{n}\right)=\frac{\vartheta_{C}}{\vartheta_{Z}\left(N_{n}\right)} \times 100$

$\mu\left(I_{n}\right)=\frac{\vartheta_{C}}{\vartheta_{Z}\left(I_{n}\right)} \times 100$

$\mu\left(A_{n}\right)=\frac{\vartheta_{C}}{\vartheta_{Z}\left(A_{n}\right)} \times 100$

The estimated energy threshold value for cluster head selection is obtained from Eqs. (19)(22), which is given as

$\vartheta_{T H V}\left(N_{n}\right)=\mu_{\left(N_{n}\right)}\left(\vartheta_{n T r}+\vartheta_{n R c}\right) \vartheta_{T r}$
$\vartheta_{T H V}\left(I_{n}\right)=\mu_{\left(I_{n}\right)}\left(\vartheta_{n T r}+\vartheta_{n R c}\right) \vartheta_{T r}$
$\vartheta_{T H V}\left(A_{n}\right)=\mu_{\left(A_{n}\right)}\left(\vartheta_{n T r}+\vartheta_{n R c}\right) \vartheta_{T r}$ 
$\vartheta_{T H V}\left(A_{n}\right), \vartheta_{T H V}\left(I_{n}\right)$ and $\vartheta_{T H V}\left(N_{n}\right)$ represent the estimated threshold values for the advanced, intermediate and normal nodes. The proposed energy threshold model is for replacing the cluster head, which minimizes the energy utilization and maximizes the node lifetime. Algorithm 1 (below) shows the energy threshold model for IoT (ETM-IoT), which aims to improve the network performance.

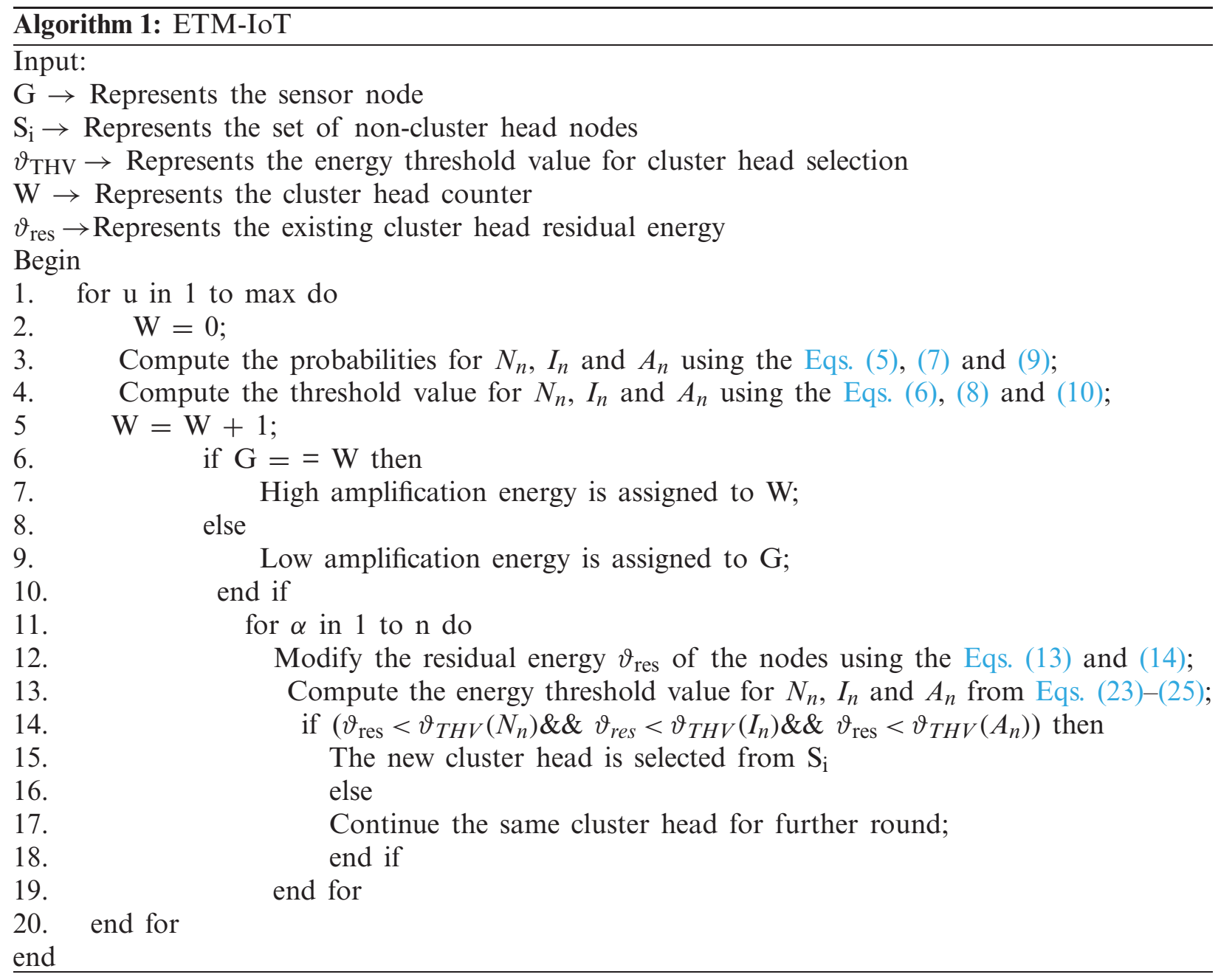

\section{Simulation Analysis}

The ETM-IoT simulation is carried out using the MATLAB. An area of $200 \times 200 \mathrm{~m}^{2}$ is considered for simulation along with 200 nodes which are deployed across it. The sink node is placed at the centre position with limitless energy.

Tab. 1 shows the parameters used in the simulated environment. The performance of the proposed ETM protocol is compared with existing protocols such as LEACH, SEP and Z-SEP. The values $\mathrm{X}$ and $\mathrm{X}$ can be varied based on different cases and the $\mathrm{Y}$ value is kept constant and is taken as 0.3 . 
CMC, 2022, vol.70, no.1

Table 1: Network simulation parameters

\begin{tabular}{ll}
\hline Parameters & Value \\
\hline Packet size $(\mathrm{n})$ & $3000 \mathrm{bits}$ \\
Distance between nodes $\left(\mathrm{d}_{0}\right)$ & $85 \mathrm{~m}$ \\
Energy consumption for data aggregation $\left(\vartheta_{\mathrm{DA}}\right)$ & $5 \mathrm{~nJ} / \mathrm{bit}$ \\
Energy consumption for free space model $\left(\vartheta_{\mathrm{fs}}\right)$ & $10 \mathrm{pJ} / \mathrm{bit} / \mathrm{m}^{2}$ \\
Energy consumption of amplifier $\left(\vartheta_{\mathrm{amp}}\right)$ & $100 \mathrm{pJ} / \mathrm{bit} / \mathrm{m}^{2}$ \\
Energy consumption for receiving $\left(\vartheta_{\mathrm{Rc}}\right)$ & $0.013 \mathrm{pJ} / \mathrm{bit} / \mathrm{m}^{4}$ \\
Total energy of the network $\left(\vartheta_{\mathrm{Tr}}, \vartheta_{\mathrm{Rc}}\right)$ & $50 \mathrm{~J}$ \\
\hline
\end{tabular}

The efficiency of the ETM-IoT routing protocol is measured using the data throughput. This is defined as the number of packets that have been successfully delivered by minimizing the packet drop ratio. In the first case, the $\mathrm{x}$ value is taken as 0.1 and $\mathrm{X}$ value is taken as 1 . In the second case, the $\mathrm{x}$ value is taken as 0.2 and $\mathrm{X}$ value is taken as 1 . The advanced nodes are taken as $10 \%$ of the network, the intermediate nodes are taken as 30\%, and the remaining nodes are normal nodes.

Figs. 4 and 5 show the network stability where the number of iterations increases until the sensor nodes eventually die. In Fig. 4, the advanced nodes are taken as $10 \%$ of the total network. It is observed that LEACH has a much shorter network lifetime compared with other routing algorithms. The LEACH protocol performs well in a homogeneous network but fails in a heterogeneous network. The proposed ETM-IoT protocol demonstrates a better performance when compared against the SEP, Z-SEP and LEACH algorithms. It shows a $26 \%$ improvement in network lifetime compared with the Z-SEP. In Fig. 5, the advanced nodes are taken as 20\% of the total network. The network lifetime using the ETM-IoT protocol increases by up to $34 \%$ when compared with Z-SEP.

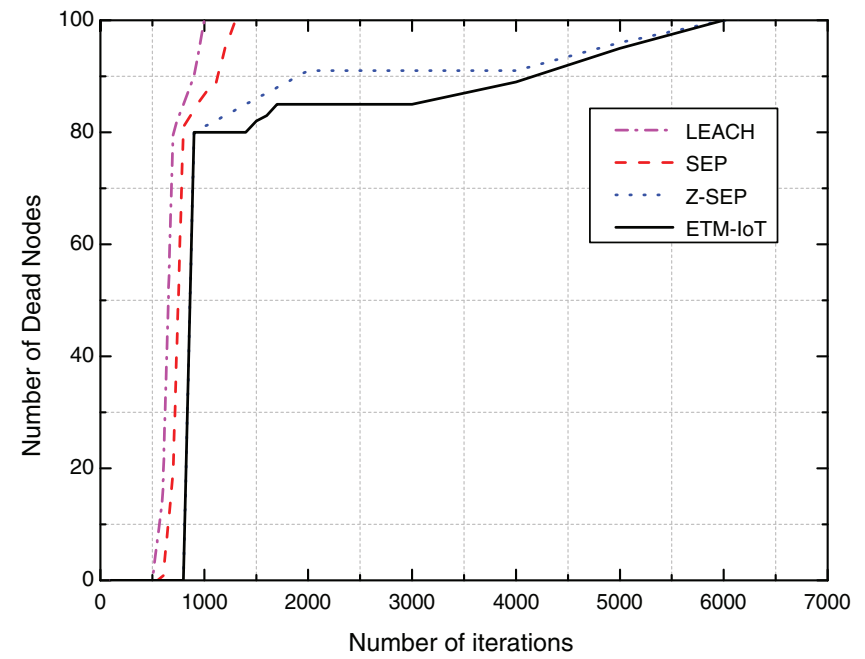

Figure 4: Network lifetime of LEACH, SEP, Z-SEP and ETM-IoT when $\mathrm{x}=01$ 


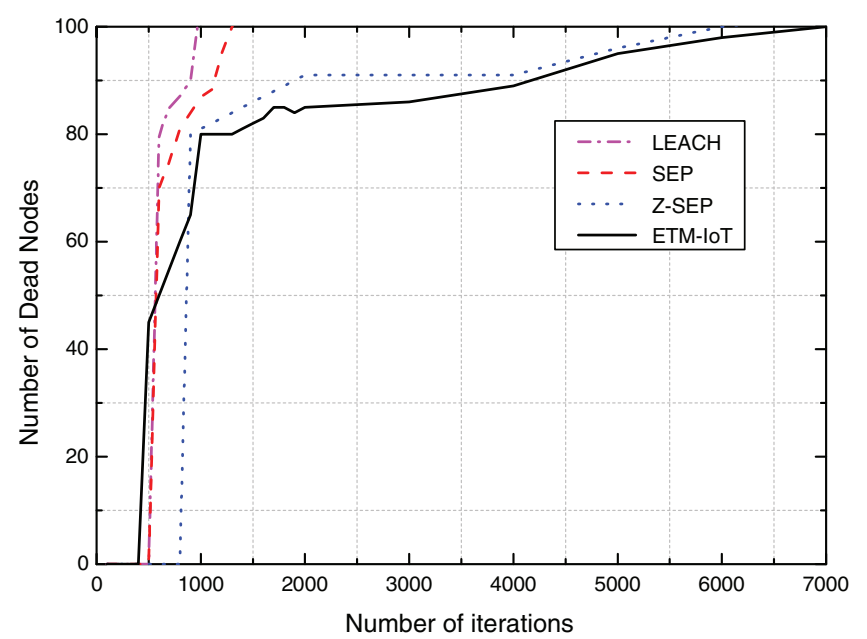

Figure 5: Network lifetime of LEACH, SEP, Z-SEP and ETM-IoT when $\mathrm{x}=0.2$

Figs. 6 and 7 show the network throughput achieved using the LEACH, SEP, Z-SEP and ETM-IoT algorithms. In the first case, with $10 \%$ of advanced nodes, the proposed routing protocol achieved the highest throughput compared with other algorithms. It recorded a $28 \%$ increase on the throughput of the Z-SEP and a 65\% improvement compared with the SEP. In the second case, with $20 \%$ of advanced nodes, the network data transfer rate also improves, with the ETM-IoT recording a 39\% improvement in throughput rate when compared with Z-SEP.

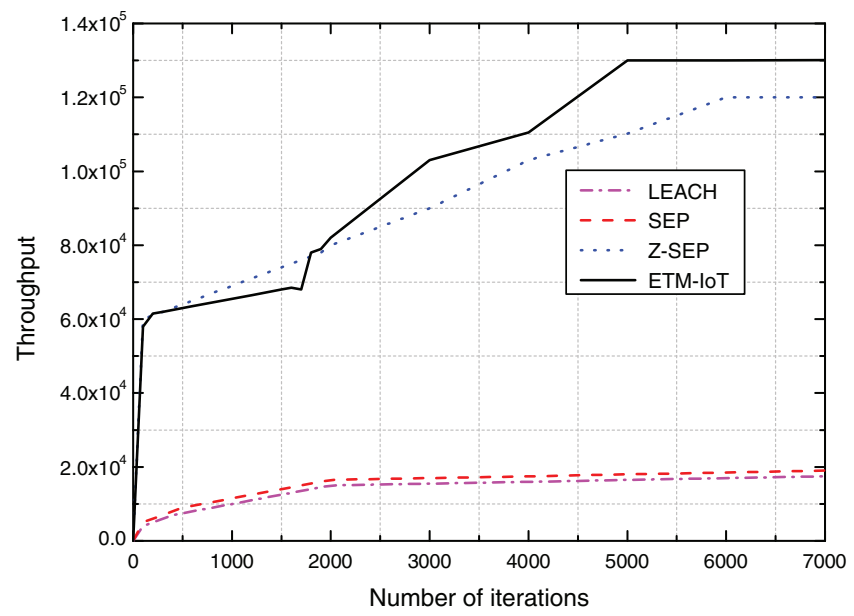

Figure 6: Network throughput of LEACH, SEP, Z-SEP and ETM-IoT when $\mathrm{x}=0.1$

Cluster heads play a major role in collecting the data from the member nodes. The cluster heads aggregate and forward data to the sink node. Figs. 8 and 9 show the formation of cluster heads in each iteration process. It is observed that the low number of cluster heads preserves the energy of the network and improves the data forwarding capability. In the first case, with 10\% advanced nodes, the ETM-IoT has fewer cluster heads when compared with the LEACH and SEP scenarios. The ETM-IoT reaches almost 10-12 cluster heads in each iteration. In the second case, with 20\% advanced nodes, the ETM-IoT reaches almost 6 to 8 cluster heads in each iteration. 


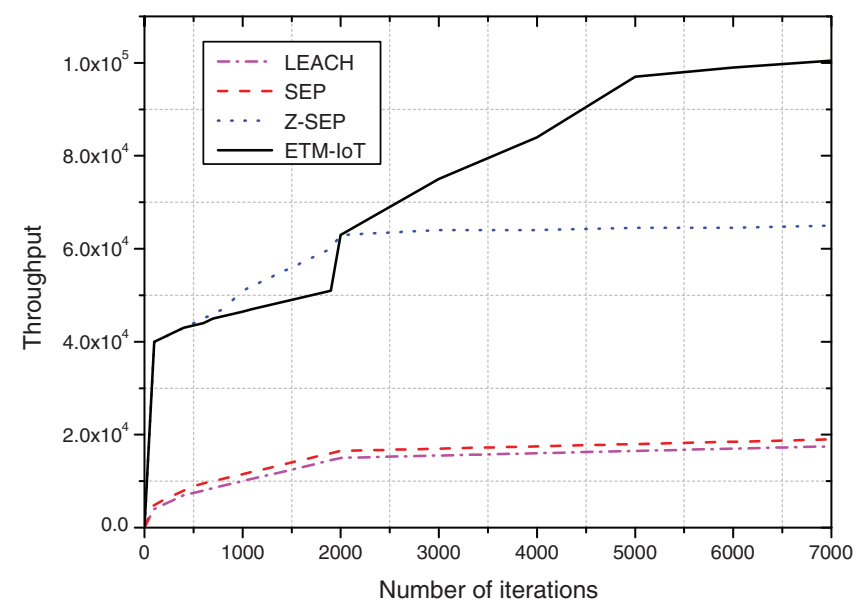

Figure 7: Network throughput of LEACH, SEP, Z-SEP and ETM-IoT when $\mathrm{x}=0.2$

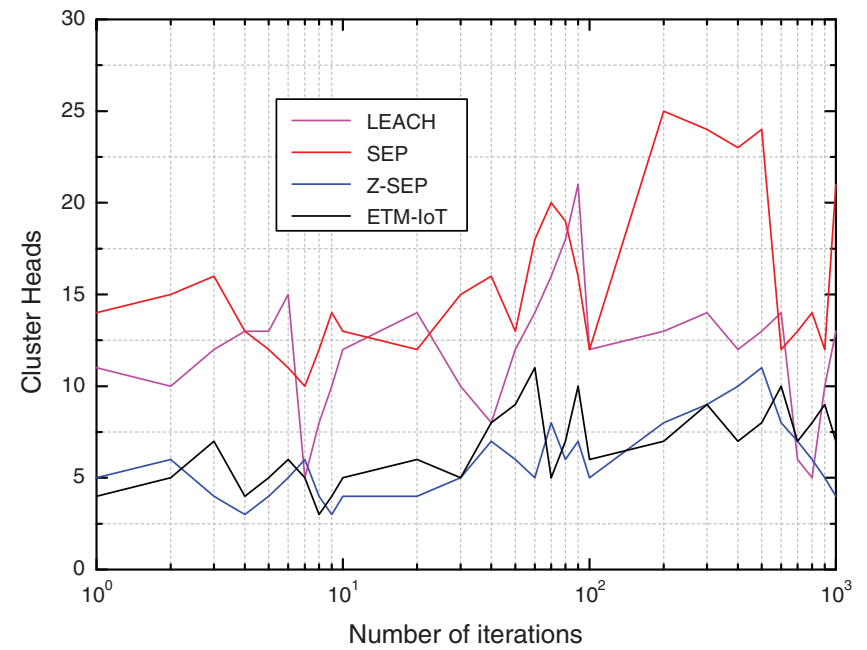

Figure 8: Cluster head count in each round when $\mathrm{x}=0.1$

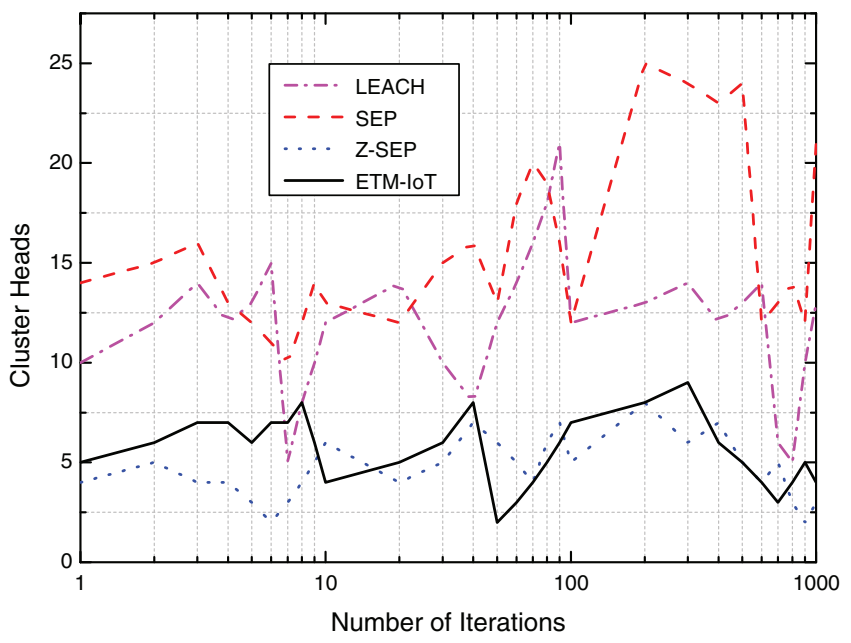

Figure 9: Cluster head count in each round when $\mathrm{x}=0.2$ 


\section{Conclusion}

This paper proposes an energy-aware model based on a threshold factor for efficient communication across the internet of things (IoT). Traditional algorithms such as the LEACH and SEP protocols are mainly used for homogeneous networks, and are ineffective when dealing with heterogonous networks. In a real-time scenario, the IoT network is composed of many sensor nodes which are limited in their energy use. Even though some objects such as mobile phones and smart watches are rechargeable, there is still a need to minimize the energy requirements of particular nodes which are limited in capacity. The proposed algorithm categorizes nodes according to three different energy threshold levels. The proposed ETM-IoT protocol distributes and regulates the energy use of the cluster heads and member nodes in the network. A simulation analysis proves the efficiency of this ETM-IoT protocol when compared with other existing protocols. The ETM-IoT protocol has the advantage of implementing real-time IoT across a large-scale environment.

Funding Statement: The authors received no specific funding for this study.

Conflicts of Interest: The authors declare that they have no conflicts of interest to report regarding the present study.

\section{References}

[1] S. Shreyas, "Context-aware energy-efficient communication for IoT sensor nodes," in Proc. 53nd ACM/EDAC/IEEE Design Automation Conf., Austin, TX, USA, pp. 1-6, 2016.

[2] G. Škulj, A. Sluga, D. Bračun and P. Butala, "Energy efficient communication based on selforganisation of IoT devices for material flow tracking," CIRP Annals, vol. 68, no. 1, pp. 495-498, 2019.

[3] H. Yanqiu, W. Yu, E. Ding and A. Garcia-Ortiz, "EPKF: Energy efficient communication schemes based on kalman filter for IoT," IEEE Internet of Things Journal, vol. 6, no. 4, pp. 6201-6211, 2019.

[4] P. Goiuri, M. Iglesias-Urkia, M. Barcelo, R. Gomez, A. Moran et al., "Fog computing based efficient IoT scheme for the industry 4.0.," in Proc. IEEE Int. Workshop of Electronics, Control, Measurement, Signals and Their Application to Mechatronics, France, pp. 1-6, 2017.

[5] A. Noura, M. Mehedi Hassan, M. Ykhlef and G. Fortino, "An efficient event matching system for semantic smart data in the internet of things (IoT) environment," Future Generation Computer Systems, vol. 95, pp. 163-174, 2019.

[6] M. Michele, F. A. Aoudia, M. Gautier, O. Berder and L. Benini, "WULora: An energy efficient IoT end-node for energy harvesting and heterogeneous communication," in Proc. Design, Automation \& Test in Europe Conf. \& Exhibition, Switzerland, pp. 1528-1533, 2017.

[7] V. Saritha and D. Nagaraju, "Energy-aware dynamic task offloading and collective task execution in mobile cloud computing," International Journal of Communication Systems, vol. 33, no. 13, pp. 27-39, 2020.

[8] Z. Xianjiao, G. Xu, X. Zheng, Y. Xiang and W. Zhou, "E-AUA: An efficient anonymous user authentication protocol for mobile IoT," IEEE Internet of Things Journal, vol. 6, no. 2, pp. 1506-1519, 2018.

[9] O. Antonino, G. Araniti, L. Militano, J. Alonso-Zarate, A. Molinaro et al., "Energy efficient IoT data collection in smart cities exploiting D2D communications," Sensors, vol. 16, no. 6, pp. 836-842, 2018.

[10] S. Liumeng, K. K. Chai, Y. Chen, J. Schormans, J. Loo et al., "Qos-aware energy-efficient cooperative scheme for cluster-based IoT systems," IEEE Systems Journal, vol. 11, no. 3, pp. 1447-1455, 2017.

[11] A. S. Fakhrul, M. G. R. Alam, R. Haw and C. S. Hong, "A system model for energy efficient green-IoT network," in Proc. 2015 Int. Conf. on Information Networking, Cambodia, pp. 177-182, 2015. 
[12] W. Kun, Y. Wang, Y. Sun, S. Guo and J. Wu, "Green industrial internet of things architecture: An energy-efficient perspective," IEEE Communications Magazine, vol. 54, no. 12, pp. 48-54, 2016.

[13] C. S. Allaoua, "Energy-efficient content-based routing in internet of things," Journal of Computer and Communications, vol. 3, no. 12, pp. 9-16, 2016.

[14] C. J. Yang, "A distributed cluster computing energy-efficient routing scheme for internet of things systems," Wireless Personal Communications, vol. 82, no. 2, pp. 757-776, 2015.

[15] E. Waleed, M. Naeem, A. Shahid, A. Anpalagan and M. Jo, "Efficient energy management for the internet of things in smart cities," IEEE Communications Magazine, vol. 55, no. 1, pp. 84-91, 2017.

[16] B. Oladayo and S. Zeadally, "Intelligent device-to-device communication in the internet of things," IEEE Systems Journal, vol. 10, no. 3, pp. 1172-1182, 2014.

[17] T. Gao, L. Zhang, Y. Gai and X. Shan, "Load-balanced cluster-based cooperative MIMO transmission for wireless sensor networks," in Proc. 4th Int. Sym. on Wireless Communication Systems, Norway, pp. 602-606, 2007.

[18] W. B. Heinzelman, A. P. Chandrakasan and H. Balakrishnan, "An application-specific protocol architecture for wireless microsensor networks," IEEE Trans. Wirel. Commun., vol. 1, no. 4, pp. 660-670, 2002.

[19] R. Saravanakumar, S. G. Susila and J. Raja, "An energy efficient cluster based node scheduling protocol for wireless sensor networks," in Proc. 10th IEEE Int. Conf. on Proc. Solid-State and Integrated Circuit Technology, California, pp. 2053-2057, 2010.

[20] G. Smaragdakis, I. Matta and A. Bestavros, "SEP: A stable election protocol for clustered heterogeneous wireless sensor networks," Boston University Computer Science Department Technical Report, vol. 1, pp. 1-11, 2004.

[21] S. Faisal, N. Javaid, A. Javaid, M. A. Khan, S. H. Bouk et al., "Z-SEP: Zonal-stable election protocol for wireless sensor networks," J. Basic Appl. Sci. Res., vol. 3, no. 5, pp. 132-139, 2013. 